\title{
Use of ultrasonic transient elastography (Fibroscan) in the assessment of hepatic focal lesion stiffness
}

\author{
Mohamed Yousef, Aisha Elsharkawy ${ }^{*}$, Mohammad El Beshlawy, Gamal Esmat, Zakaria Salama \\ Department of Endemic Medicine and Hepatology, Faculty of Medicine, Cairo University, Cairo, Egypt \\ Email: *a_m_sharkawy@yahoo.com
}

Received 27 January 2013; revised 25 March 2013; accepted 1 May 2013

Copyright (C 2013 Mohamed Yousef et al. This is an open access article distributed under the Creative Commons Attribution License, which permits unrestricted use, distribution, and reproduction in any medium, provided the original work is properly cited.

\begin{abstract}
Background and Aim: Hepatic focal lesions differ in their tissue composition and in the degree of stiffness, so our aim was to evaluate the role of Ultrasonic Transient Elastography (Fibroscan) in the measurement of hepatic focal lesions stiffness in order to differentiate hepatocellular carcinoma (HCC) focal lesions from other non HCC focal lesions. Methods: The study was conducted on 34 patients with hepatic focal lesion(s) in the right lobe located near the liver surface and more than or equal $4 \mathrm{~cm}$ in diameter, detected by imaging studies and diagnosed by $\mathrm{CT}$ and histopathology. Stiffness over the focal lesions was measured by the fibroscan. Results: The median value of stiffness was 72.5 kPa over HCC focal lesions, 17.2 kPa over lymphoma focal lesions, $6.5 \mathrm{kPa}$ over metastatic focal lesions and $10.5 \mathrm{kPa}$ over the sarcoidosis focal lesion. Conclusions: Hepatocellular carcinoma focal lesions are much stiffer than lymphoma, metastasis or sarcoidosis focal lesions. Fibroscan may be a useful non-invasive method in the prediction of hepatocellular carcinoma in the future.
\end{abstract}

Keywords: Hepatic Focal Lesions; Hepatocellular Carcinoma; Fibroscan; Stiffness

\section{INTRODUCTION}

The increased use of radiologic imaging, particularly ultrasound examination, has led to much more frequent identification of nodules in the liver [1]. Hepatocellular carcinoma (HCC) is the fifth most common malignancy in the world [2] complicating liver cirrhosis in most cases. Its incidence is increasing worldwide ranging between 3\% and 9\% annually [3]. In Egypt, HCC was reported to account for about $4.7 \%$ of chronic liver disease

${ }^{*}$ Corresponding author.
(CLD) patients [4]. Incidence of HCC in Egypt is currently increasing, which may be the result of a shift in the relative importance of hepatitis $\mathrm{B}$ virus and hepatitis $C$ virus as primary risk factors [5]. In the setting of a patient with known hepatitis B or cirrhosis of other etiology, a mass found incidentally or on screening ultrasound has a high likelihood of being HCC [6].

Hepatic metastases are the most frequent malignancies in the liver. The incidence of hepatic metastases is approximately $40 \%$ in patients with colorectal cancer [7].

Primary hepatic lymphomas represent rare neoplasms but secondary hepatic lymphomas are much more frequent and represent disseminated disease [8].

Sarcoidosis may appear in the liver as multiple space occupying hepatic lesions worrisome for metastatic disease and a needle biopsy is important for the diagnosis [9].

Transient elastography (Fibroscan, Echosens, Paris, France) is a novel rapid, noninvasive, reproducible method for measuring liver stiffness. Tissue stiffness is directly proportional to the square of shear wave velocity: the stiffer the tissue, the faster the shear wave propagates [10]. Theoretically, the elastography can also assess stiffness of hepatic tumors. However, the diameter of the tumor must be comparable to the region of interest (ROI) of transient elastography, which is currently between 25 and $65 \mathrm{~mm}$ from the surface [11].

The aim of this study was to evaluate the use of Fibroscan in the measurement of the stiffness over different types of hepatic focal lesions in order to differentiate HCC focal lesions from other non HCC focal lesions.

\section{MATERIALS AND METHODS}

\subsection{Patients}

The study was conducted on 34 patients with hepatic focal lesion(s) in the right lobe located near the liver surface and more than or equal $4 \mathrm{~cm}$ in diameter, de- 
tected by imaging studies (as abdominal ultrasound or triphasic CT). The study protocol was approved by the institutional review board. Written informed consent was given by each patient.

\subsection{Diagnosis of Hepatic Focal Lesions}

It was made by dynamic CT, where intrahepatic nodules with hyperattenuation in the arterial phase and with washout in the late phase were considered as definite HCC [12]. Hepatic focal lesions were diagnosed as lymphoma, metastasis or sarcoidosis by histopathological assessment of the lesion after taking an ultrasound guided biopsy. Accordingly patients were classified into 2 main groups: 1-HCC hepatic focal lesions (26 patients) and 2-Non HCC hepatic focal lesions (4 lymphoma, 3 metastasis, 1 sarcoidosis).

\subsection{Evaluation of Tumor Stiffness Using Fibroscan}

It was performed using the fibroscan device (Echosens, Paris, France) located at the Kasr Al Aini Viral Hepatitis Center, which consists of $5 \mathrm{MHz}$ ultrasound transducer probe mounted on the axis of a vibrator. TE measures liver stiffness in a volume that approximates a cylinder of $1 \mathrm{~cm}$ wide and $4 \mathrm{~cm}$ long, between 25 and $65 \mathrm{~mm}$ below skin surface, and so the measurement depth or ROI (region of interest) is set between 25 and $65 \mathrm{~mm}$ from the surface. Tumor stiffness was measured according to Masuzaki et al. [11] as follows. First, by using B-mode ultrasound, we search for the optimal right intercostal position where the focal lesion can be viewed as large in diameter and as near to the surface as possible while keeping the ultrasound probe and body surface at right angles. Then at the same position and angle, the vibrator is applied and the stiffness is measured according to the manufacturer's instruction. The median value of ten successful acquisitions expressed in kilopascal $(\mathrm{kPa})$ is calculated and kept as representative of focal lesion stiffness.

\subsection{Statistical Analysis}

Patients' data were analyzed using SAS 9.2 for windows 7. Quantitative variables were expressed by median (the median is the "middle value" in a list), IQR, mean (the mean is the arithmetic average of a set of values) and SD (Standard deviation), compared using t-student test and Mann-Whitney U test were used when appropriate. Pearson and Spearman's rho were used for correlating quantitative variables when appropriate. Qualitative variables were expressed by numbers (Frequency) and percent. Proportions were analyzed using Fisher's exact test and Chi-square test when appropriate. ROC-curve was ap- plied, calculating the AUC, sensitivity and specificity for the tests used. Cut-off values were calculated. Univariate prediction model was performed for calculating predicted probabilities, and data were graphically represented. $\mathrm{p}$-value was considered to be significant if less than 0.05 .

\section{RESULTS}

The present study was conducted on 34 patients with different types of hepatic focal lesions and according to their diagnosis they were classified into 26 patient with HCC hepatic focal lesions and eight patients with non HCC hepatic focal lesions (4 lymphoma, 3 metastasis, 1 sarcoidosis). Patients with HCC were significantly older than those with non HCC focal lesions (mean age $57 \pm$ 5.7 versus $42 \pm 15.7$ years, $p=0.03$ ) with higher male predominance in the HCC group $(92.3 \%)$ as shown in Table 1. Regarding the laboratory profile of patients in the HCC group, the median value of AFP was statistically significant and expectedly higher than in the non HCC group (89.5 versus 7.5, $\mathrm{p}=0.000$ ).

The median value of stiffness was $72.5 \mathrm{kPa}$ over HCC focal lesions and $9.4 \mathrm{kPa}$ over non HCC focal lesions, this difference was statistically highly significant ( $\mathrm{p}=$ 0.000) (Figure 1). The median value of stiffness was $17.2 \mathrm{kPa}$ over lymphoma hepatic focal lesions, $6.5 \mathrm{kPa}$ over metastatic focal lesions and $10.5 \mathrm{kPa}$ over the case of sarcoidosis focal lesion as shown in Table 2 and Figure 2.

In 13 patients out of the 26 patient of the HCC group, we were able to measure the stiffness in an area away from the hepatic focal lesion. The comparison between the stiffness over the lesion and that away from the lesion shows that there was a positive relationship between the stiffness over the lesion and the stiffness away from the lesion in HCC patients with $r=0.707$ and $p$ value of 0.007 .

Also there was a positive relationship between the stiffness over the HCC lesion and the level of AFP with $r$ $=0.047$ and $p$ value of 0.014 : the patients with very high AFP had generally higher stiffness values.

The predicted probability to HCC by measuring the stiffness over the focal lesion has OR: 1.33, 95\% CI: $0.99-1.78$ and p-value of 0.056 (which is approaching significant) the overall accuracy was $94.1 \%$, sensitivity: 96.2\%, specificity: $87.5 \%$, PPV (positive predictive value): $96.2 \%$ and NPV (negative predictive value): $87.5 \%$ as

Table 1. Demographic data of the studied population.

\begin{tabular}{cccc}
\hline & HCC (\%) & Non HCC (\%) & p value \\
\hline $\begin{array}{c}\text { AGE } \\
\text { (Mean } \pm \text { SD) }\end{array}$ & $57 \pm 5.7$ & $42 \pm 15.7$ & 0.03 \\
Male & $92.3 \%$ & $50 \%$ & \\
Female & $7.7 \%$ & $50 \%$ & 0.01 \\
\hline
\end{tabular}




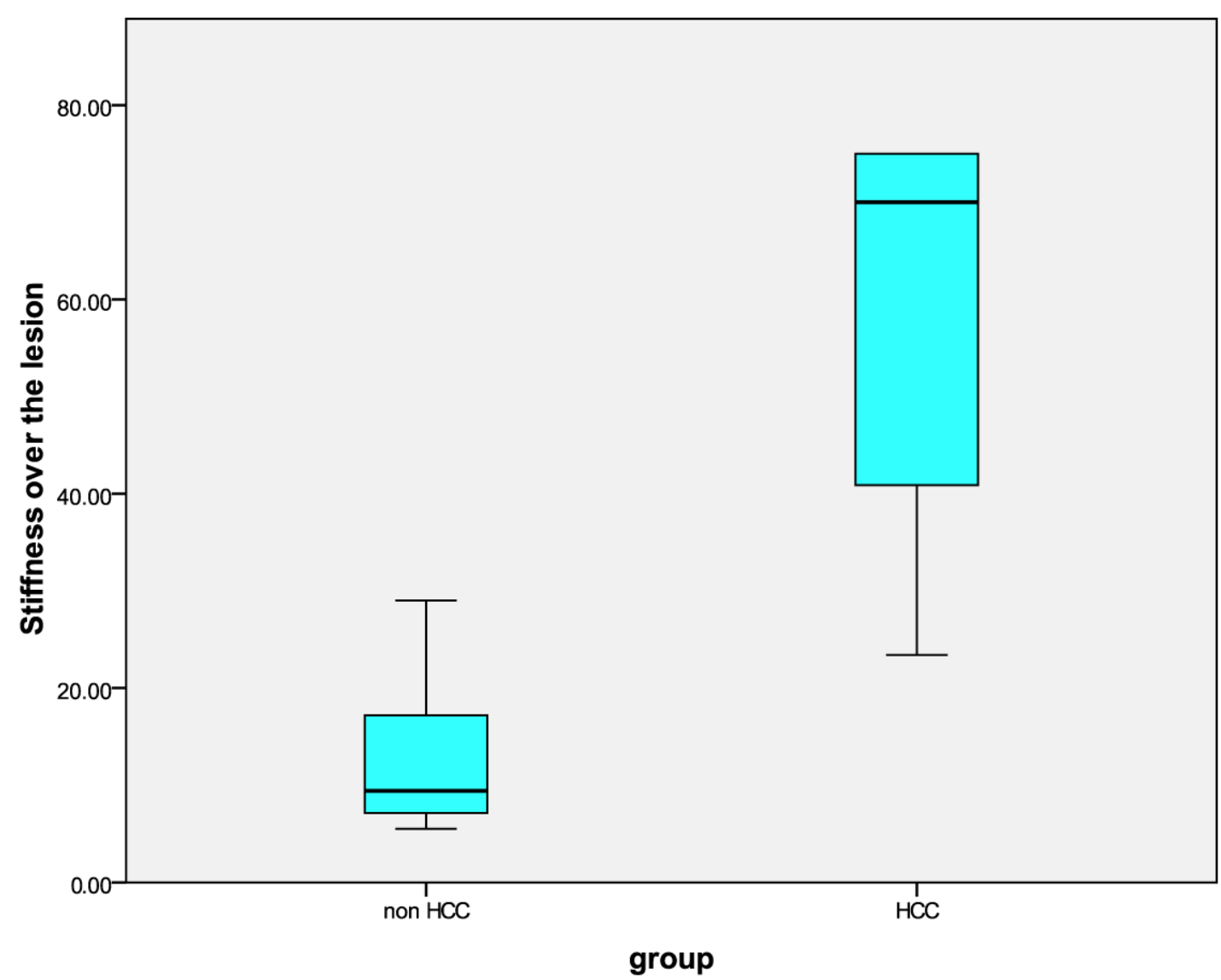

Figure 1. Comparing stiffness over focal lesions in both groups.

Table 2. Stiffness over the hepatic focal lesions.

\begin{tabular}{ccc}
\hline Variable & number & Median $\mathrm{kPa}($ minimum - maximum) \\
\hline HCC & 26 & $72.5(23-75)$ \\
Lymphoma & 4 & $17.2(8-29)$ \\
Metastasis & 3 & $6.5(5.5-7.8)$ \\
Sarcoidosis & 1 & 10.5 \\
\hline
\end{tabular}

shown in Figure 3.

\section{DISCUSSION}

The frequent detection of hepatic focal lesions and uncertainty about their nature using imaging modalities (without interventional and risky tissue biopsy) constitute a major problem in clinical practice especially in Egypt where chronic liver disease constitutes a national threat to its population. So our aim was to evaluate the role of Ultrasonic Transient Elastography (Fibroscan) in the measurement of hepatic focal lesions stiffness so that it can be used as a potential diagnostic modality in discrimination of HCC focal lesion from other non HCC focal lesion.

In the present study, we had 26 cases with HCC and eight cases with non HCC hepatic focal lesions: 3 cases were diagnosed as metastatic adenocarcinomas (8.8\% of the total number of cases), 4 cases were diagnosed as lymphoma (11.7\% of the total number of cases) and one case with focal lesion was diagnosed as sarcoidosis. This high incidence of HCC in our patients is mainly attributed to the high prevalence of HCV-related liver disease and cirrhosis in Egypt; the latter being a well-established risk factor to the development of HCC and this matches with Barletta et al. [13] who stated that HCC often arises as a complication of long standing cirrhosis mainly induced by chronic HBV or HCV infection.

Another explanation to this higher percentage of HCC in our patients, can be attributed to the technical requirement for the measurement of tumor stiffness by Fibroscan which requires large lesion in the right hepatic lobe near the surface excluding large number of patients with small multiple focal lesions (a substantial group of patients with secondaries) that could-otherwise-be included in the study.

In the present study, we found that hepatocellular carcinoma focal lesions are much stiffer than lymphoma, metastasis or sarcoidosis focal lesions ( $p$ value of 0.00 ) which could allow us in the future to modify this device in order to predict HCC especially if we can come to a cut-off value and these results can be reproduced in fur- 


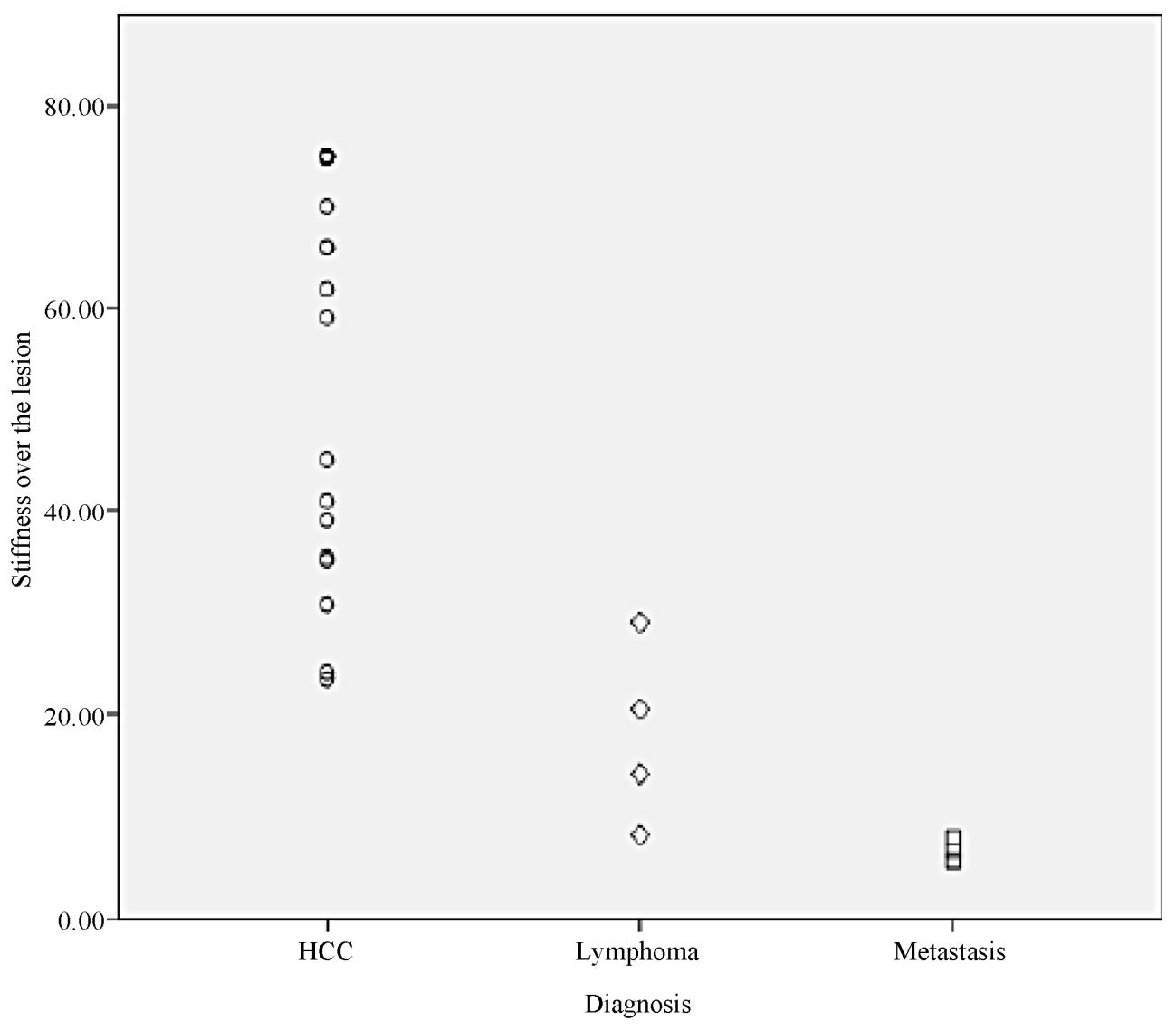

Figure 2. Distribution of focal lesions stiffness values in the whole studied population.

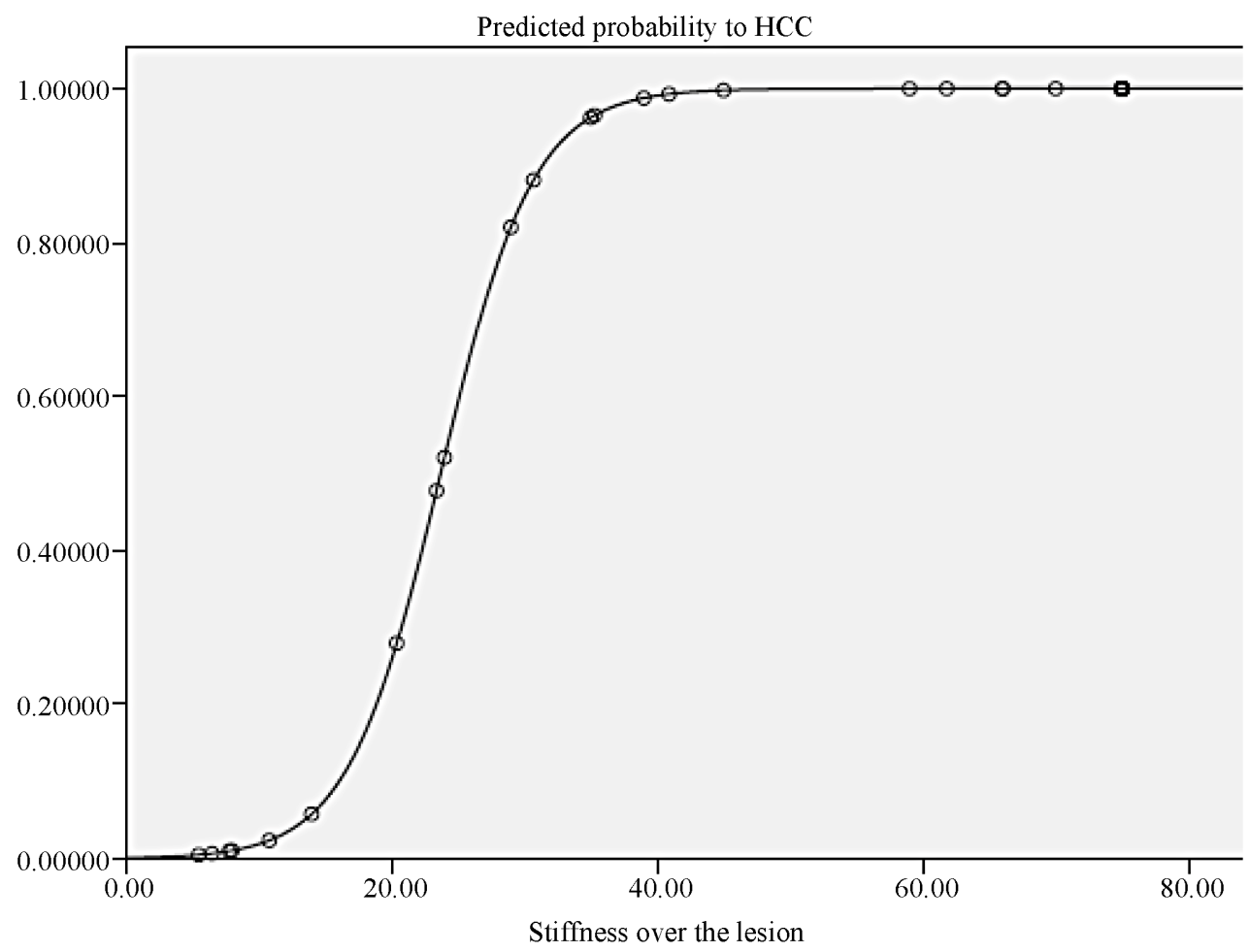

Figure 3. Prediction model to HCC by fibroscan data (stiffness over the lesion) for (HCC \& nonHCC group). 
ther works.

By comparing our results to Masuzaki et al. study [11] which was conducted on 40 patients, 17 with HCC, 6 with cholangiocarcinoma, 16 with metastatic tumors (mostly adenocarcinoma), and one with malignant lymphoma; the difference in the median value of stiffness in the HCC group (72.5 kPa in our study versus $55 \mathrm{kPa}$ in Masuzaki study) may be attributed to the difference in the number of patients in each study (26 patients in our study versus 17 patients in Masuzaki's study, stage of the tumor and the underlying risk factors for development of HCC (difference in percentage of cirrhosis).

Also the difference in the results in the metastasis group between the present study and Masuzaki's study, may be attributed to the larger number of patients with metastatic tumors included in their study and the variety of the primary tumors as submandibular gland, esophagus, stomach, rhabdomyosarcoma, gall bladder, breast, colon and ovarian tumors while all our patients with metastasis had adenocarcinoma of the colon and this variety in the nature of primary tumor may affect the stiffness of secondaries.

Our study showed that the predicted probability of HCC by using stiffness measurement over the focal lesion was approaching significance (p value: 0.05 ) and so we can say that the more the stiffness over the hepatic focal lesion the more is the predicted probability of being HCC and this probability reaches the highest value when the stiffness over the hepatic focal lesion is higher than $40 \mathrm{kPa}$.

But the present study has some limitations. First, the small number of patients in our study is attributed to the technical difficulties in the measurements of stiffness of hepatic focal lesions which require large lesion more than $4 \mathrm{~cm}$ in diameter in the right hepatic lobe, near the surface and in patients with no ascites. Second, we gathered all patients with hepatic focal lesions other than HCC in one group due to the very limited number of patients in the non HCC group (eight cases) in spite of their different nature and finally we didn't have cholangiocarcioma focal lesions in this study.

In 13 patients out of 26 patients with HCC, we could measure the stiffness in an area of the liver away from the lesion (non tumoral area) and we found that the more is the stiffness over the non tumoral liver tissue, the more is the stiffness over the HCC focal lesion for the same patient with $r$ value of 0.707 and $p$ value of 0.007 .

\section{CONCLUSION}

Hepatocellular carcinoma focal lesions are much stiffer than lymphoma, metastasis or sarcoidosis focal lesions. Fibroscan may be a useful non-invasive method in the prediction of hepatocellular carcinoma in the future.

\section{ACKNOWLEDGEMENTS}

We thank Professor Dr. Ayman Yosry, the head of the Endemic Medicine Department in Kasr Alaini, Professor Dr. Nabeel Elkady, Professor of Endemic Medicine and Ultrasonography in Kasr Alaini and Professor Dr. Ashraf Omar, the head of the HCC clinic in Kasr Alaini, for their help to complete this work.

\section{REFERENCES}

[1] Bosch, F.X., Ribes, J., Diaz, M. and Cleries, R. (2004) Primary liver cancer: Worldwide incidence and trends. Gastroenterology, 127, S5-S16.

[2] El-Serag, H.B. (2002) Hepatocellular carcinoma: An epidemiologic view. Journal of Clinical Gastroenterology, 35, S72-S78. doi:10.1097/00004836-200211002-00002

[3] Velazquez, R.E., Rodriguez, M., Navascues, C.A., Linares, A., Perez, R., Sotorrios, N.G., Martinez, I. and Rodrigo, L. (2003) Prospective analysis of risk factors for hepatocellular carcinoma in patients with liver cirrhosis. Hepatology, 37, 520-527. doi:10.1053/jhep.2003.50093

[4] El-Zayadi, A.R., Abaza, H., Shawky, S., Mohamed, M.K., Selim, O.E. and Badran, H.M. (2001) Prevalence and epidemiological features of hepatocellular carcinoma in Egypt-A single center experience. Hepatology Research, 19, 170-179. doi:10.1016/S1386-6346(00)00105-4

[5] El-Zayadi, A.R., Badran, H.M., Barakat, E.M., Attia, M.E., Shawky, S., Mohamed, M.K., Selim, O. and Saeid, A. (2005) Hepatocellular carcinoma in Egypt: A single center study over a decade. World Journal of Gastroenterology, 11, 5193-5198.

[6] Bruix, J. and Sherman, M. (2005) Practice Guidelines Committee, American Association for the Study of Liver Diseases. Management of hepatocellular carcinoma. $\mathrm{He}$ patology, 42, 1208-1236.

[7] Coenegrachts, K. (2009) Magnetic resonance imaging of the liver: New imaging strategies for evaluating focal liver lesions. World Journal of Radiology, 1, 72-85.

[8] Longerich, T., Schirmacher, P., Dienes, H., Stein, H. and Loddenkemper, C. (2006) Malignant lymphomas of the liver: New diagnostic algorithms. Der Pathologe, 27, 263-272.

[9] Powell, J.L, Cunill, E.S., Gajewski, W.H. and Novotny, D.B. (2005) Sarcoidosis mimicking recurrent endometrial cancer. Gynecologic Oncology, 99, 770-773.

[10] Castera, L., Vergniol, J., Foucher, J., Le Bail, B., Chanteloup, E., Haaser, M., Darriet, M., Couzigou, P. and De Lédinghen, V. (2005) Prospective comparison of transient elastography, Fibrotest, APRI, and liver biopsy for the assessment of fibrosis in chronic hepatitis C. Gastroenterology, 128, 343-350. doi:10.1053/j.gastro.2004.11.018

[11] Masuzaki, R., Tateishi, R., Yoshida, H., Sato, T., Ohki, T., Goto, T., Hideo, Y., Shinpei, S., Yosuke, S., Hitoshi, I., Shuichiro, S., Takao, K. and Masao, O. (2007) Assessing liver tumor stiffness by transient elastography. Journal of Hepatology, 1, 394-397.

[12] Torzilli, G., Procopio, F., Cimino, M., Del Fabbro, D., 
Palmisano, A., Donadon, M. and Montorsi, M. (2010) Anatomical segmental and subsegmental resection of the liver for hepatocellular carcinoma: A new approach by means of ultrasound-guided vessel compression. Annals of Surgery, 251, 229-235.

\section{ABBREVIATIONS}

AFP: Alpha-fetoprotein;

CLD: Chronic liver disease;
[13] Barletta, E., Tinessa, V. and Daniele, B. (2005) Screening of hepatocellular carcinoma: Role of the alpha-fetoprotein (AFP) and ultrasonography. Recenti Progressi in $\mathrm{Me}$ dicina, 96, 295-259.

HCC: Hepatocellular carcinoma;

ROI: Region of interest;

CT: Computed tomography. 